\title{
Multiscale Fabrication of Multiple Proteins and Topographical Structures by Combining Capillary Force Lithography and Microscope Projection Photolithography
}

\section{Supporting Information}

\author{
Keon Woo Kwon, ${ }^{\dagger, \S}$ Jong-Cheol Choi ${ }^{\S}$ Kahp-Yang Suh ${ }^{*, \dagger,}$ and Junsang Doh ${ }^{*, \S, ~}$, I
}

${ }^{\dagger}$ School of Mechanical and Aerospace Engineering and ${ }^{\star}$ World Class University Program on Multiscale Mechanical Design, Seoul National University, Seoul, 151-742, Korea

${ }^{\S}$ Department of Mechanical Engineering, $\|_{\text {School of Interdisciplinary Bioscience and Bioengineering (I-Bio) and World }}$ Class University Program Division of Integrative Biosciences and Biotechnology (IBB), Pohang University of Science and Technology (POSTECH) San 31, Hyoja-dong, Nam-Gu, Pohang, Gyeongbuk, 790-784, Korea

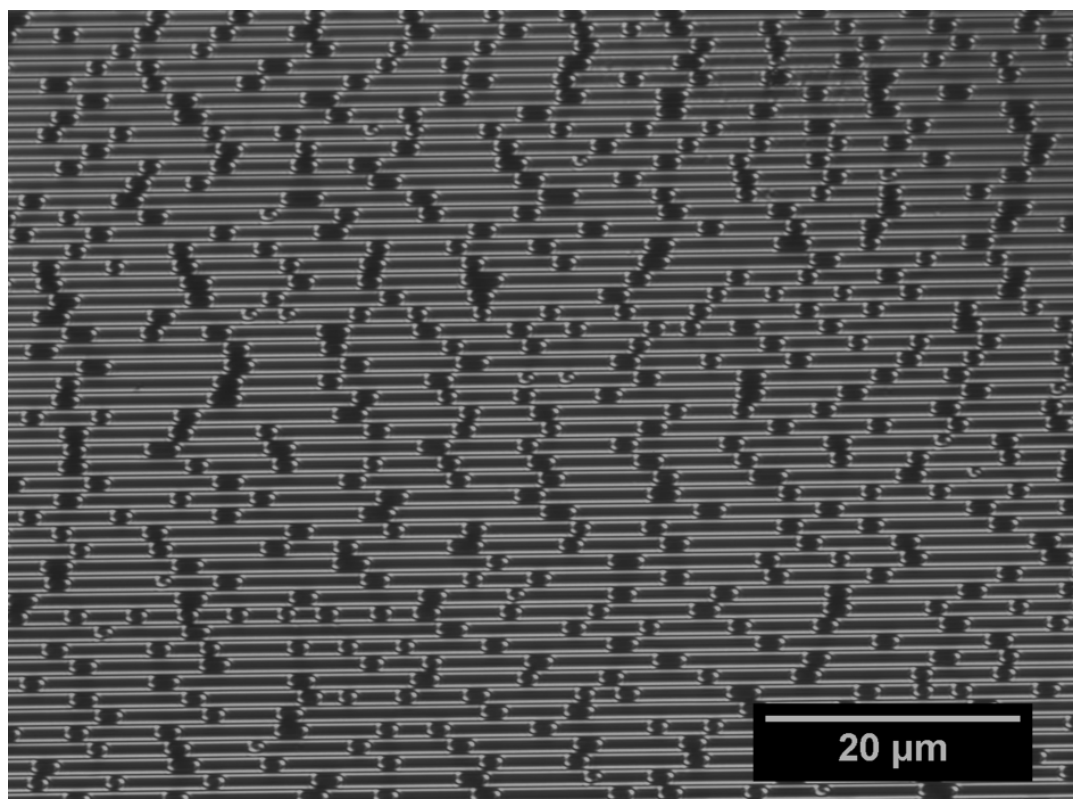

Supporting Figure 1. A representative optical microscope image of disconnected $700 \mathrm{~nm}$ ridges by dewetting. PDMP thin films cast from $8 \%$ solution was used.

Supporting Video 1. Time-lapse video of SW480 cells attached on $16 \mu \mathrm{m}$ micro-grooves with perpendicular nano-grooves. 